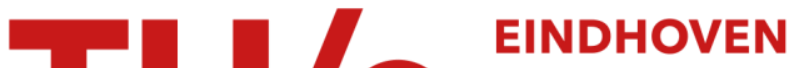 UNIVERSITY OF TECHNOLOGY
}

\section{Band structure and density of states effects in Co-based magnetic tunnel junctions}

\section{Citation for published version (APA):}

LeClair, P., Kohlhepp, J. T., van de Vin, C. H., Wieldraaijer, H., Swagten, H. J. M., de Jonge, W. J. M., Davis, A. H., MacLaren, J. M., Moodera, J. S., \& Jansen, R. (2002). Band structure and density of states effects in Cobased magnetic tunnel junctions. Physical Review Letters, 88(10), [107201].

https://doi.org/10.1103/PhysRevLett.88.107201, https://doi.org/10.1103/PhysRevLett.88.107201

DOI:

10.1103/PhysRevLett.88.107201

10.1103/PhysRevLett.88.107201

Document status and date:

Published: 01/01/2002

\section{Document Version:}

Publisher's PDF, also known as Version of Record (includes final page, issue and volume numbers)

\section{Please check the document version of this publication:}

- A submitted manuscript is the version of the article upon submission and before peer-review. There can be important differences between the submitted version and the official published version of record. People interested in the research are advised to contact the author for the final version of the publication, or visit the $\mathrm{DOI}$ to the publisher's website.

- The final author version and the galley proof are versions of the publication after peer review.

- The final published version features the final layout of the paper including the volume, issue and page numbers.

Link to publication

\section{General rights}

Copyright and moral rights for the publications made accessible in the public portal are retained by the authors and/or other copyright owners and it is a condition of accessing publications that users recognise and abide by the legal requirements associated with these rights.

- Users may download and print one copy of any publication from the public portal for the purpose of private study or research.

- You may not further distribute the material or use it for any profit-making activity or commercial gain

- You may freely distribute the URL identifying the publication in the public portal.

If the publication is distributed under the terms of Article 25fa of the Dutch Copyright Act, indicated by the "Taverne" license above, please follow below link for the End User Agreement:

www.tue.nl/taverne

Take down policy

If you believe that this document breaches copyright please contact us at:

openaccess@tue.nl

providing details and we will investigate your claim. 


\title{
Band Structure and Density of States Effects in Co-Based Magnetic Tunnel Junctions
}

\author{
P. LeClair, ${ }^{1}$ J. T. Kohlhepp, ${ }^{1}$ C. H. van de Vin, ${ }^{1}$ H. Wieldraaijer, ${ }^{1}$ H. J. M. Swagten, ${ }^{1}$ W. J. M. de Jonge, ${ }^{1}$ \\ A. H. Davis, ${ }^{2}$ J. M. MacLaren, ${ }^{2}$ J. S. Moodera, ${ }^{3}$ and R. Jansen ${ }^{3,4}$ \\ ${ }^{1}$ Department of Applied Physics, Eindhoven University of Technology, P.O. Box 513, 5600 MB Eindhoven, The Netherlands \\ ${ }^{2}$ Department of Physics, Tulane University, New Orleans, Louisiana 70118 \\ ${ }^{3}$ Francis Bitter Magnet Laboratory, Massachusetts Institute of Technology, Cambridge, Massachusetts 02139 \\ ${ }^{4} \mathrm{MESA}^{+}$Research Institute, University of Twente, 7500 AE Enschede, The Netherlands
}

(Received 4 July 2001; published 20 February 2002)

\begin{abstract}
Utilizing $\mathrm{Co} / \mathrm{Al}_{2} \mathrm{O}_{3} / \mathrm{Co}$ magnetic tunnel junctions with Co electrodes of different crystalline phases, a clear relationship between electrode crystal structure and junction transport properties is presented. For junctions with one fcc(111) textured and one polycrystalline (polyphase and polydirectional) Co electrode, a strong asymmetry is observed in the magnetotransport properties, while when both electrodes are polycrystalline the magnetotransport is essentially symmetric. These observations are successfully explained within a model based on ballistic tunneling between the calculated band structures (density of states) of fcc-Co and hcp-Co.
\end{abstract}

DOI: $10.1103 /$ PhysRevLett.88.107201

One of the most basic and essential aspects of magnetic tunnel junctions (MTJs) [1] is the role of the electronic structure in determining MTJ properties. The sensitivity of tunnel current to the electronic structure of the tunneling electrodes has been well established theoretically for some time [2-4]. Experimentally, there have been few conclusive observations of the electronic structure of a normal (i.e., not superconducting or a semimetal [5]) metal in tunneling experiments, despite extensive efforts [6-8]. Spin polarized tunneling in superconductorinsulator-ferromagnet junctions [9], for example, can only reveal the spin polarization of the participating density of states (DOS) (at the Fermi level) of electrons tunneling from the ferromagnet. More recently, De Teresa et al. [10] have used half-metallic $\mathrm{La}_{0.7} \mathrm{Sr}_{0.3} \mathrm{MnO}_{3}$ (LSMO) in $\mathrm{Co} / \mathrm{SrTiO}_{3} / \mathrm{LSMO}$ junctions to analyze the sign and magnitude of the Co spin polarization as a function of bias, pointing out the importance of participating tunneling states. Yuasa et al. [11] have also recently shown a dependence of the tunneling spin polarization on crystallographic orientation for epitaxial Fe electrodes. Still, few examples of a clear experimental correlation between the conductance properties and the electronic structure of normal metal electrodes with a clear theoretical analysis have been identified, particularly for magnetic junctions.

The difficulties in observing band structure or density of states effects in normal metal junctions (or MTJs) are numerous. One limitation is that one can only hope to see band or DOS features for those bands that contribute to the tunnel conductance. These bands and the DOS they generate are (for the $\mathrm{Al}_{2} \mathrm{O}_{3}$ barriers utilized) believed to be limited to highly dispersive bands whose states at finite $k$ are $s$ hybridized $[3,4,6,9,12]$. A second complication is the presence of many conductance (i.e., $d I / d V$ ) contributions, such as normal elastic tunneling and inelastic excitations (phonons, magnons), which may obscure electronic structure features, but which may be essentially
PACS numbers: 73.40.Gk, 75.70.-i, 85.30.Mn, 85.70.Kh

eliminated with careful analysis [6]. A third complication is the extreme difficulty in the theoretical analysis of these structures, particularly MTJs. A last, and perhaps most fatal, complication is the need for tunneling electrodes with a known physical and electronic structure which can be modulated in order to convincingly compare theory and experiment.

In this Letter, we prepare $\mathrm{Co} / \mathrm{Al}_{2} \mathrm{O}_{3} / \mathrm{Co}$ MTJs with differently textured $\mathrm{Co}$ layers, viz., highly textured fcc(111)-Co and polycrystalline-Co, in order to realize a model system in which to study the relation between electronic and crystal structure and MTJ properties. Using only Co, but in different crystalline phases (and hence with different electronic structure), crucially allows us to focus only on changes in the electronic and crystal structure. By a careful analysis of the conductance-voltage $(d I / d V-V)$ and magnetoresistance-voltage characteristics, in combination with ${ }^{59} \mathrm{Co}$ nuclear magnetic resonance (NMR) to directly investigate local physical structure, we show experimentally that junctions with electrodes of two different crystalline phases show obviously asymmetric transport characteristics. The observed asymmetries can be qualitatively explained by a model developed by us [13-15] based on ballistic tunneling between the calculated band structures (and resulting DOS) of fcc-Co and hcp-Co. Furthermore, these experimental and theoretical results may explain several related observations of similar conductance asymmetries [8,16-18].

Ferromagnetic tunnel junctions were prepared by UHV $\mathrm{dc} / \mathrm{rf}$ magnetron sputtering; the details of this fabrication process have been described in $[19,20]$. The tunnel magnetoresistance (TMR) structures used consisted of $\mathrm{Si}(001) /$ $\mathrm{SiO}_{2} /$ buffer/Co $d_{\mathrm{Co}} / \mathrm{Al}_{2} \mathrm{O}_{3} / \mathrm{Co} 150 \AA / \mathrm{Al} 30 \AA$ postannealed in a magnetic field at $200{ }^{\circ} \mathrm{C}$. For these experiments, the buffer was either Ta $50 \AA /$ Co $70 \AA / F e M n$ $100 \AA$ ("FeMn-based buffer") or only Ta $50 \AA$ ("Ta buffer"). Junction resistances or differential resistances 
$\left(d V / d I \equiv G^{-1}\right.$, where $d I / d V \equiv G$ is the conductance) were measured using standard $d c$ or $a c$ lock-in techniques. In all cases, the bottom electrode was biased positively for $V>0$.

In order to prove that Co layers grown on FeMn-based and Ta-based buffers have a different physical structure, we performed ${ }^{59} \mathrm{Co}$ NMR measurements on separately grown $\mathrm{Si} / \mathrm{SiO}_{2} / \mathrm{Ta} 50 \AA / \mathrm{Co} d d_{\mathrm{Co}} / \mathrm{Al}_{2} \mathrm{O}_{3}$ (Ta-based), $\mathrm{Si} /$ $\mathrm{SiO} 2 / \mathrm{Ta} 50 \AA / \mathrm{Cu} 70 \AA / \mathrm{FeMn} 100 \AA / \mathrm{Co} d_{\mathrm{CO}} / \mathrm{Al}_{2} \mathrm{O}_{3}$ (FeMn-based), and $\mathrm{Al}_{2} \mathrm{O}_{3} / \mathrm{Co} 150 \AA$ (to represent the top electrode) structures, sputtered under identical conditions [21]. The NMR experiments were performed at $1.5 \mathrm{~K}$ in zero applied field, as described in [22]. NMR measurements give information on the distribution hyperfine fields at the Co nuclei, from which it is possible to distinguish Co atoms in different structural environments (e.g., fcc, hcp, bcc) and to determine their relative amounts $[22,23]$.

Figure 1 shows the resulting ${ }^{59} \mathrm{Co}$ NMR intensity as a function of frequency for FeMn-based structures (Fig. 1a) with $d_{\mathrm{Co}}=35,50,80 \AA, \mathrm{Al}_{2} \mathrm{O}_{3} / \mathrm{Co} / \mathrm{Al}$ structures with $d_{\mathrm{Co}}=80 \AA$, and for a Ta-based structure with $d_{\mathrm{Co}}=$ $50 \AA$ (Fig. 1b). The vertical lines indicate the frequencies for bulk hcp (magnetization $\|$ or $\perp$ to the $c$ axis) and fcc Co. Focusing on the FeMn-based structures, (Fig. 1a), a strong line is observed at the frequency corresponding to fcc-Co for $d_{\mathrm{Co}}=50 \AA$ and $80 \AA$, with little intensity at the hcp-Co positions or intervening regions (corresponding to stacking faults $[22,23])$. For the $35 \AA$, the dominant line is still fcc-Co, but considerable intensity exists at the hcp-Co and stacking fault positions. However, from 35-80 $\AA$ the stacking fault intensity does not increase. From this, we may conclude that Co growth begins with a considerable amount of stacking faults, but then grows in a more ordered fcc structure, with presumably the purest fcc-Co present at the uppermost surface. Thus, even the

(a) FeMn based

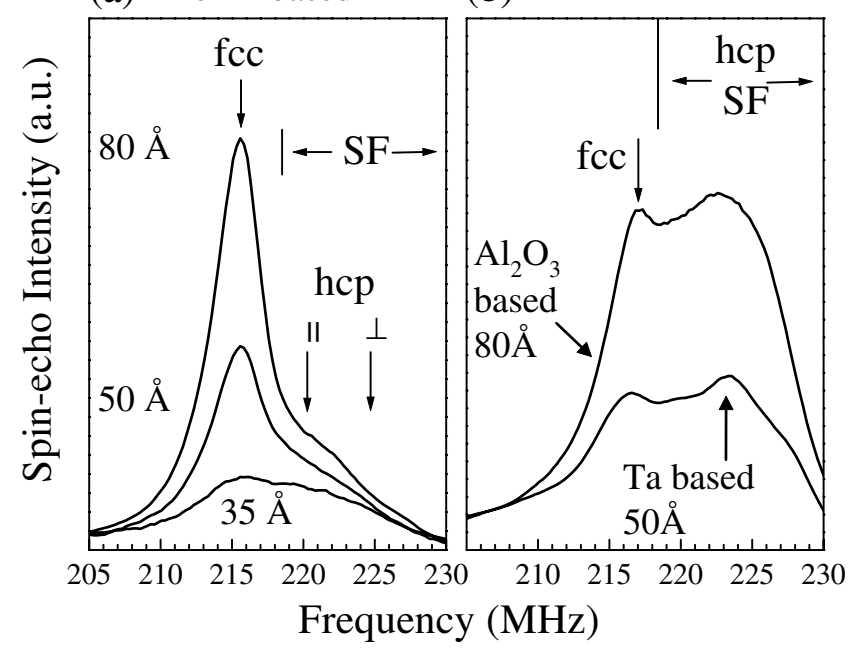

FIG. 1. ${ }^{59} \mathrm{Co}$ NMR spectra at $1.5 \mathrm{~K}$ in zero applied field for (a) FeMn-based structures with varying $d_{\mathrm{Co}}$ (see text) and (b) $\mathrm{Ta} / \mathrm{Co} / \mathrm{Al}_{2} \mathrm{O}_{3}$ and $\mathrm{Al}_{2} \mathrm{O}_{3} / \mathrm{Co} / \mathrm{Al}$ structures.
$35 \AA$ Co layer has predominantly fcc-Co stacking for the topmost layers adjacent to the barrier. Combining the NMR data with $\mathrm{x}$-ray diffraction measurements indicates that the Co layers with FeMn-based buffers are (111) textured fcc-Co.

Figure $1 \mathrm{~b}$ shows the NMR intensity spectrum for representative Ta-based and $\mathrm{Al}_{2} \mathrm{O}_{3}$-based samples. No significant Co thickness dependence was observed, indicating that the growth mode is the same for all thicknesses. For either the Ta-based samples or those grown on $\mathrm{Al}_{2} \mathrm{O}_{3}$, intensity is observed for both hcp positions as well as the fcc position and all intermediate stacking fault positions. From this, we may conclude that Co grows on Ta or $\mathrm{Al}_{2} \mathrm{O}_{3}$ in a random, polycrystalline and polyphase manner $[22,23]$ (which we refer to as "poly-Co" for simplicity), in contrast to the fcc(111)-textured Co in FeMn-based structures. Thus, the FeMn-based and Ta-based MTJs prepared for this study may be clearly identified as asymmetric fcc(111)-Co/ $\mathrm{Al}_{2} \mathrm{O}_{3} /$ poly-Co and symmetric poly$\mathrm{Co} / \mathrm{Al}_{2} \mathrm{O}_{3} /$ poly-Co junctions, respectively. We must caution that, although the FeMn-based junctions produce strongly fcc(111)-textured bottom electrodes, they contain a fraction of $\mathrm{Co}$ atoms which remains in a non-fcc environment.

One may anticipate that these structural differences lead to differences in band structure and DOS, and, hence, differences in transport properties. From symmetry, one expects that a physically asymmetric junction results in transport which is asymmetric with respect to bias. Figure 2a shows the conductance-voltage $[d I / d V(V) \equiv$ $G(V)]$ characteristics, normalized at $V=0$, for parallel Co magnetizations at $5 \mathrm{~K}$ for two fcc(111)-Co $d_{\mathrm{Co}} /$ $\mathrm{Al}_{2} \mathrm{O}_{3} /$ poly-Co junctions with $d_{\mathrm{Co}}=35,50 \AA$ and a poly-Co/ $\mathrm{Al}_{2} \mathrm{O}_{3} /$ poly-Co junction. The fcc(111)-Co/ $\mathrm{Al}_{2} \mathrm{O}_{3} /$ poly-Co junctions show an obvious conductance asymmetry, with most notably a local minimum at

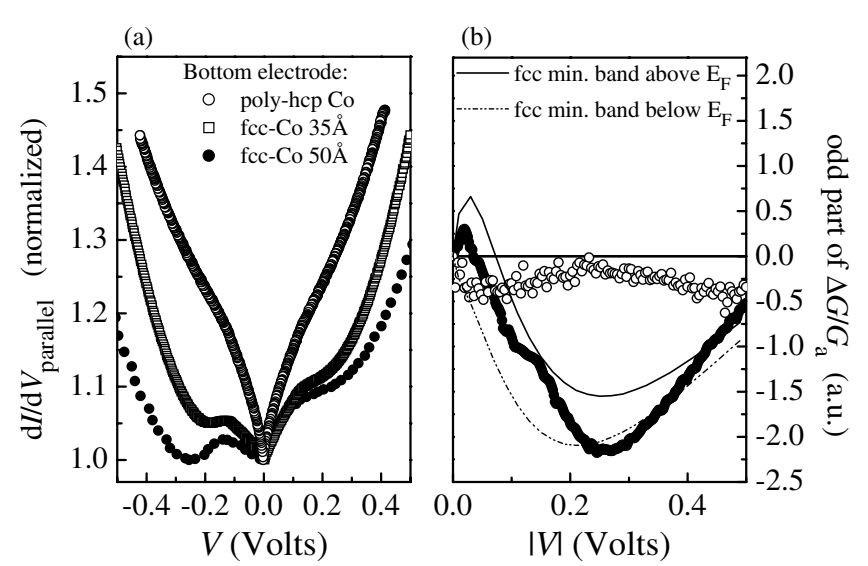

FIG. 2. (a) Conductance-voltage $(d I / d V-V)$ characteristics, normalized at zero bias, for fcc(111)-Co $35 \AA, 50 \AA / \mathrm{Al}_{2} \mathrm{O}_{3} /$ poly-Co, and poly-Co/ $\mathrm{Al}_{2} \mathrm{O}_{3} /$ poly-Co junctions, for parallel magnetization alignments at $5 \mathrm{~K}$. (b) Odd part of $\Delta G$ / $G_{a}$ vs voltage. Experimental data at $5 \mathrm{~K}$ (points), and calculated curves (lines, $\times 5$ ) for a fcc-Co/I/hcp-Co junction (see text). 
$\sim-0.25 \mathrm{~V}$ and a slight "shoulder" at the same positive voltage, only for parallel magnetizations. Parenthetically, we note that the asymmetry was also present for intentionally over- or underoxidized barriers. We will return to the explanation of these unusual features shortly. The poly-Co/ $\mathrm{Al}_{2} \mathrm{O}_{3} /$ poly-Co junctions show almost perfectly symmetric behavior for both magnetization orientations, with a parabolic background and a low-voltage linear contribution, with no apparent thickness dependence. For both types of junctions, the former parabolic portion can be understood in terms of regular elastic tunneling [6], whereas the linear contribution is consistent with magnon-assisted tunneling [16]. However, the local minimum and shoulder observed for fcc(111)-Co/ $\mathrm{Al}_{2} \mathrm{O}_{3} /$ poly-Co junctions cannot be explained by either of these mechanisms. The striking difference between junctions where only the crystalline phase of the bottom electrode was intentionally altered suggests that the electronic structure and spin-dependent DOS of the fcc-Co structure must be responsible.

If the DOS and band structure differences between the two Co electrodes are responsible, one may expect the bias dependence of the TMR to be altered as well. Although the TMR was generally lower for poly-Co/ $\mathrm{Al}_{2} \mathrm{O}_{3} /$ poly-Co structures, this criterion is very susceptible to slight differences in preparation conditions. The normalized differential TMR-voltage $\left[\Delta G / G_{\text {ap }}(V)\right.$, normalized at $V=0$ ] characteristics, though generally nearly identical for many junctions [20], suffer from a strong contribution by inelastic excitations [16] which may mask the underlying electronic structure effects. The main inelastic excitation contribution is due to magnon excitations in the electrodes [16], which give a contribution symmetric in applied bias. If we assume as a rough approximation that the magnon excitation spectra for poly-Co and fcc(111)-Co are similar, by plotting the odd part of the differential TMR-voltage behavior, the magnonassisted conductance contributions cancel, and we may be primarily sensitive to only electronic structure (or selfenergy) effects [6]. For any excitations within the barrier (e.g., phonons), this assumption is very well justified [6]. We emphasize that this sort of analysis is crucial for comparison with theory, effectively eliminating those contributions which are difficult to incorporate theoretically. Figure $2 \mathrm{~b}$ shows the odd portion of $\Delta G / G_{a}$ [i.e., $\Delta G / G_{\text {ap }}(V>0)-\Delta G / G_{\text {ap }}(V<0)$ ] as a function of voltage, a measure of the asymmetry in the bias dependence of the differential TMR, $\Delta G / G_{\text {ap }}$ [24]. For poly-Co/ $\mathrm{Al}_{2} \mathrm{O}_{3} /$ poly-Co junctions, almost no asymmetry is present, as expected for nominally identical electrodes. However, for fcc(111)-Co/ $\mathrm{Al}_{2} \mathrm{O}_{3} /$ poly-Co junctions, there is an obvious strong minimum, corresponding to the same voltage where the local minimum and shoulder features were seen in the $d I / d V(V)$ curves. This further suggests that the electrode DOS and band structure may play a key role. Since the differential TMR more strongly decreases at the position of the shoulder and local minimum, one expects that either the minority DOS increases or the majority DOS decreases. As we will show, the dip and shoulder features found in $d I / d V(V)$ can be accounted for theoretically by a combination of band structure and DOS arguments, incorporated into a ballistic model of tunneling [13-15].

The key input for the model used [13-15] is the spinresolved DOS of the electrodes. The calculated DOS for fcc-Co [13] shows that the total DOS is negatively polarized at $E_{F}$, but that the $s$-DOS is positively polarized. This suggests that states with $s$ character dominate the tunneling with these barriers $[4,9,12]$. Calculations by us show that the most dispersive bands, corresponding to those which have previously been identified as the source of the tunneling conductance, exhibit significant $s$ hybridization. Since these are hybridized states, every contribution to the $s$ character of the band is accompanied by a contribution to the $d$ character from the same state (and, to a smaller extent, the $p$ character). For $\mathrm{Fe}, \mathrm{Co}$, and $\mathrm{Ni}$, in each case there is only a single band with significant $s$ character near $E_{F}$. Therefore, the $s$-DOS emerges as a good marker for the behavior of the conductance $(d I / d V)$ in this bias range. To obtain the $s$-hybridized partial DOS, the DOS and band structures have been calculated with the layer-KKR (Korringa-Kohn-Rostoker) method [25]. For simplicity, we use hcp(0001) bands to model the poly-Co electrode, since we are looking primarily at features of the fcc DOS, and the hcp s-hybridized bands are relatively featureless in any case. Although it has been previously shown that tunneling is extremely interface sensitive $[19,26]$, we expect that the Co band structure and DOS at the barrier interface, though certainly altered, will strongly resemble the bulk, at least with regard to the general features one may observe [27].

We first observe that the fcc(111) DOS for states with $s$ character above and below $E_{F}$ has several key features which may explain the underlying tendency for $d I / d V$ to dip in fcc(111)-Co/ $\mathrm{Al}_{2} \mathrm{O}_{3} /$ poly-Co junctions. The fcc-Co $s$-DOS is known to exhibit two sharp peaks at about $0.4 \mathrm{eV}$ above and below $E_{F}$ [13]. DOS peaks imply localized states that have a reduced tunneling probability $[9,12,13]$, and, hence, a smaller contribution to the conductance. For the fcc(111)-Co/ $\mathrm{Al}_{2} \mathrm{O}_{3} /$ poly-Co structures, note that in the experimental configuration electrons flow from hcp to fcc bands for positive bias, and fcc to hep bands for negative bias. For negative bias, tunneling into the poly-Co electrode, biasing includes occupied states from below $E_{F}$ in the fcc electrode. Since the fcc states below $E_{F}$ are increasingly localized as bias increases toward $0.4 \mathrm{eV}, d I / d V$ is suppressed, resulting in a $d I / d V$ dip. For tunneling into the fcc electrode at positive bias, we expect the same tendency of $d I / d V$ to decrease, because now the similarly localized states above $E_{F}$ are participating. However, for positive bias, an additional effect enters the picture. In the fcc-Co band structure, there is an unoccupied, but very dispersive, minority band which begins just above $E_{F}$ [13]. Since it is above $E_{F}$, it only 
participates for tunneling into the fcc-Co electrode from the poly-Co electrode (positive bias). This highly dispersive band contributes spin down states, thus decreasing the TMR, but augmenting $d I / d V$, since it leads to an overall increase in available states. The emergence of this band thus partially suppresses the tendency of $d I / d V$ to dip for $V>0$. As a result, the dip for positive bias in $d I / d V$ appears as a shoulder. Since this band participates only for positive bias, it suppresses the TMR only for positive bias, leading to a minimum in the odd part of the differential TMR. Interestingly, earlier work with $\mathrm{Cu}$ dusted MTJs $[19,20]$ showed that dusting of the fcc electrode suppresses the dip in $d I / d V$, which in hindsight may further indicate that the dips are fcc-Co specific features.

Figure $2 \mathrm{~b}$ shows the odd part of the differential TMR calculated for a fcc-Co/I/hcp-Co MTJ using the model described in Refs. $[14,15]$. For the poly-Co/I/fcc-Co structure, the small peak at small biases comes from the aforementioned minority fcc-Co band beginning just above $E_{F}$. When this minority fcc-Co band is artificially shifted to begin below $E_{F}$ instead of above $E_{F}$, the small peak disappears. Thus, appearance of the minority band beginning just above $E_{F}$ in fcc-Co is clearly responsible for this feature. The overall tendency for the odd part of the differential TMR to increase at low biases is sharply suppressed when this minority band emerges for slightly larger biases. Since the appearance of this band strongly increases the contribution of minority states, the odd part of the differential TMR therefore decreases. Thus, the small peak in the odd part of the TMR is directly related to the unoccupied minority band of the fcc-Co structure, and the localized part of the fcc-Co $s$-DOS is responsible for the deep minimum in the odd part of the differential TMR and the tendency of $d I / d V$ to dip at $|V| \sim 0.25 \mathrm{~V}$. Although the qualitative agreement is excellent, the quantitative agreement is not-calculated values are roughly a factor 5 too small (see Fig. 2b) - probably due to the neglect of conductance contributions which are not symmetric in bias (e.g., differing magnon spectra in the two electrodes) and the relative simplicity of the model used. However, this is as yet a common problem when modeling MTJs. We note parenthetically that the calculated bias dependence for hcp-Co/I/hcp-Co junctions is completely symmetric, and the odd part of the differential TMR is thus identically zero. The small experimentally observed odd part for the poly-Co/I/poly-Co junctions, which resembles that of the poly-Co/I/fcc-Co junction, is probably due to the fact that the poly-Co electrodes contain some fcc(111) crystallites, and in slightly different amounts for the top and bottom electrodes. Finally, we note that these results may explain several similar observations in literature $[8,17,18]$.

In conclusion, we have shown a clear relation between the electronic and physical structure of the magnetic electrodes and the magnetotransport properties of MTJs. The asymmetric magnetotransport properties may be understood by considering the itinerant electron bands in the two crystalline phases of $\mathrm{Co}$, as determined from firstprinciples calculations, and incorporated into a model of ballistic tunneling.

P. LeClair is supported by the Netherlands technology foundation STW, and work at Tulane was supported by DARPA Grant No. MDA 972-97-1-003.

[1] J. S. Moodera, L. R. Kinder, T. M. Wong, and R. Meservey, Phys. Rev. Lett. 74, 3273 (1995).

[2] J. A. Appelbaum and W. F. Brinkman, Phys. Rev. 186, 464 (1969).

[3] S. Zhang and P. M. Levy, Eur. Phys. J. B 10, 599 (1999).

[4] I. I. Oleinik, E. Y. Tsymbal, and D. G. Pettifor, Phys. Rev. B 62, 3952 (2000).

[5] A. I. Khachaturov, A. A. Galkin, E. Hatta, and V. M. Svistunov, Low Temp. Phys. 26, 827 (2000).

[6] E. L. Wolf in Principles of Electron Tunneling Spectroscopy, (Oxford University Press, London, 1985), Chap. 8.

[7] R. C. Jaklevic and J. Lambe, Surf. Sci. 37, 922 (1973).

[8] J. M. Rowell, J. Appl. Phys. 40, 1211 (1969).

[9] R. Meservey and P. M. Tedrow, Phys. Rep. 238, 173 (1994).

[10] J. M. D. Teresa et al., Science 286, 507 (1999).

[11] S. Yuasa et al., Europhys. Lett. 52, 344 (2000).

[12] W.H. Butler, X. G. Zhang, T.C. Schulthess, and J. M. MacLaren, Phys. Rev. B 63, 054416 (2001).

[13] A. Davis, Ph. D. thesis, (Tulane University, New Orleans, LA, 2000).

[14] A. H. Davis, J. M. MacLaren, and P. LeClair, J. Appl. Phys. 89, 7567 (2001).

[15] A. H. Davis and J. M. MacLaren, J. Appl. Phys. 87, 5224 (2000).

[16] S. Zhang, P. M. Levy, A. C. Marley, and S. S. P. Parkin, Phys. Rev. Lett. 79, 3744 (1997).

[17] J. S. Moodera, J. Nowak, and R. J. M. van de Veerdonk, Phys. Rev. Lett. 80, 2941 (1998).

[18] W. Oepts et al., J. Appl. Phys. 89, 8038 (2001).

[19] P. LeClair et al., Phys. Rev. Lett. 84, 2933 (2000).

[20] P. LeClair et al., Appl. Phys. Lett. 76, 3783 (2000).

[21] In this case, a $\mathrm{Cu}$ layer was used rather than $\mathrm{Co}$ as a polycrystalline seed layer for the FeMn layer, but this has no effect on the resulting texture.

[22] W. J. M. de Jonge, H. A. M. de Gronckel, and K. Kopinga, in Ultrathin Magnetic Structures II, edited by B. Heinrich and J. A. C. Bland (Springer-Verlag, Berlin, 1994), p. 279.

[23] P. C. Riedi, T. Thomson, and G. J. Tomka, in Handbook of Magnetic Materials, edited by K. H. J. Buschow (Elsevier, Amsterdam, 1999), Vol. 12, pp. 97-258.

[24] This is not the same as $\Delta R / R_{p}$ for finite bias.

[25] J. M. MacLaren et al., Comput. Phys. Commun. 60, 365 (1990).

[26] P. LeClair et al., Phys. Rev. Lett. 86, 1066 (2001).

[27] We expect interface states to be localized, and, hence, not contribute significantly to the tunnel current. 